\title{
EFFECT OF SELF-MANAGEMENT EDUCATION VERSUS QUADRICEPS STRENGTHENING EXERCISES ON PAIN AND FUNCTION IN PATIENTS WITH KNEE OSTEOARTHRITIS
}

original paper

(1) University School of Physical Education in Wroclaw

DOI: https://doi.org/10.5114/hm.2018.76081

\section{OLUFUNMILAYO R. EGWU ${ }^{1}$, OLUSOLA O. AYANNIYI ${ }^{2}$, BABATUNDE O.A. ADEGOKE ${ }^{2}$, OLADAPO M. OLAGBEGI ${ }^{3}$, OMOYEMI O. OGWUMIKE ${ }^{2}$, ADESOLA C. ODOLE ${ }^{2}$}

${ }^{1}$ Department of Physiotherapy, Barau Dikko Teaching Hospital, Kaduna, Nigeria

${ }^{2}$ Department of Physiotherapy, College of Medicine, University of Ibadan, Ibadan, Nigeria

${ }^{3}$ Department of Physiotherapy, School of Health Sciences, University of KwaZulu-Natal, Durban, South Africa

\section{ABSTRACT}

Purpose. To investigate and compare the effects of 6-week self-management education (SME) and quadriceps strengthening exercises (QSE) on pain and disability in individuals with knee osteoarthritis (OA).

Methods. A total of 79 (13 males, 66 females) consecutive patients with knee OA were randomised into SME and QSE groups. The SME group were taught modules of self-management once a week for 6 weeks, while the QSE group had supervised QSE thrice a week for 6 weeks. Pain intensity and physical function were assessed with the Visual Analogue Scale and Ibadan Knee/Hip Osteoarthritis Outcome Measure (IKHOAM) at baseline, week 6, and at the follow-up time points of the $1^{\text {st }}, 2^{\text {nd }}$ and $3^{\text {rd }}$ months.

Results. The effects of both interventions were comparable $(p>0.005)$ during intervention and follow-up. During intervention, in both groups, pain intensity significantly decreased $(p<0.001$, effect size $=0.603)$ and IKHOAM scores improved $(p<0.001$, effect size $=0.540)$. There were significant time by group interaction effects during follow-up as pain intensity $(p<0.001$, effect size $=0.085)$ did not change in the QSE group but further decreased in the SME group, IKHOAM scores $(p=0.005$, effect size $=0.053$ ) remained the same in the SME group while it decreased in the QSE group with respect to the end of intervention $\left(6^{\text {th }}\right.$ week).

Conclusions. Supervised QSE and SME are both effective in reducing pain and disability in knee OA but improvements in the outcomes are better sustained with SME.

Key words: knee osteoarthritis, self-management education, quadriceps strengthening exercises

\section{Introduction}

Osteoarthritis (OA) is a major musculoskeletal challenge characterised by slow degradation of cartilage, pain, and increasing chronic disability [1]. It is a frequent cause of morbidity, functional limitations, and loss of autonomy in the second half of human life $[1,2]$. Internationally, OA affects about $24 \%$ of the general population, with symptomatic OA observed in $9.6 \%$ of men and $18 \%$ of women aged 60 years and above; the disease also occurs in about $80 \%$ of people aged over 65 years in high-income countries [3].
The majority of patients with OA are managed in primary care, and the prevalence of knee OA is such that simple interventions which are effective in the community setting are necessary [4]. There is no known modality for reversing the progression of knee OA; it can only be managed by ameliorating its symptoms [4]. Treatment of OA is aimed at reducing pain and disability to improve function and quality of life [5-7]. International treatment guidelines have recommended a combination of pharmacological and non-pharmacological therapies [8, 9]; surgical intervention is only indicated when the aforementioned approaches

Correspondence address: Dr Oladapo Michael Olagbegi, Department of Physiotherapy, School of Health Sciences, University of KwaZulu-Natal, Westville Campus, Durban, 4001, South Africa, e-mail: dapoolagbegi2015@gmail.com

Received: August 18, 2017

Accepted for publication: January 29, 2018

Citation: Egwu OR, Ayanniyi OO, Adegoke BOA, Olagbegi OM, Ogwumike OO, Odole AC. Effect of self-management education versus quadriceps strengthening exercises on pain and function in patients with knee osteoarthritis. Hum Mov. 2018; 19(3):64-74; doi: https://doi.org/10.5114/hm.2018.76081. 
fail [10]. However, drug treatments such as nonsteroidal anti-inflammatory drugs (NSAID), aimed at relieving pain, are often limited by gastrointestinal side effects [11]; cyclooxygenase-2 (COX-2) inhibitors, used to salvage these side effects, are also associated with adverse cardiovascular incidents such as myocardial infarction [12]. Moreover, little or no evidence supports disease-modifying OA drugs such as glucosamine and chondroitin, which intensifies interest in non-pharmacologic treatments for OA $[13,14]$.

Non-pharmacological approaches to knee OA management include education, self-management, exercise, appliances (walking sticks, insoles, and knee bracing), acupuncture, telephone contact, physical therapy, and weight reduction [8-10]. McAlindon et al. [9], in a systematic review, identified modalities such as self-management education (SME), strengthening exercises, aerobic/fitness exercises (land- or water-based), and weight management as core aspects of managing OA. It was also suggested that these modalities could easily be adapted for self-care, considering the chronic nature of knee OA [9].

Chronic knee OA reportedly leads to marked weakening of the quadriceps femoris muscle, which functionally act as extensor and stabilizer of the knee [15]. Quadriceps muscle weakness results in its declined ability to reduce load across the knee joint, which increases joint stress; it may thus play a role in the aetiology and progression of knee OA [16, 17]. Therefore, ageing and atrophy of this muscle largely contribute to knee pain and functional impairment in knee OA [16]. A recent systematic review by Fransen et al. [18] concluded that land-based therapeutic exercises generally provided benefits in terms of reduced knee pain and improved physical function and quality of life among people with knee OA. Another systematic review, by Lange et al. [5], specifically provides strong evidence in support of quadriceps strengthening as a modality for reducing pain and disability in patients with knee OA. This indicates that well-planned quadriceps strengthening exercises (QSE) for patients with knee OA can achieve pain relief and functional recovery of the muscle [19].

SME has become a popular component of management in several chronic conditions including arthritis and has been listed alongside therapeutic exercises as the mainstay of non-pharmacologic treatment of knee OA [5, 7, 20]. It is aimed at achieving more than just the provision of information to increase knowledge, which makes it different from the traditional patient education programmes. The purpose of self-management programmes is to change health behaviour and health status by teaching patients to identify and solve problems, set goals, and plan actions [21]. A SME programme specific for knee OA was found to be effective in reducing pain and disability, as well as improving the health-related quality of life in patients with knee OA [22, 23].

A meta-analysis [24] concluded that exercise needs to be performed 3 times a week for optimal benefits. In spite of these benefits, exercise seems more expensive and demanding in terms of hospital attendance (to the patient) and health care resources (to the government) than SME. Studies comparing the effects of SME and supervised QSE are rather scarce. However, comparison of the 2 modalities may provide useful evidence on their relative efficacy in order to give an informed appraisal of the choice of therapy for patients with knee OA, especially in developing nations with limited resources. This study was therefore designed to compare and evaluate the effects of SME and QSE programmes on pain and physical function in individuals with knee OA as well as their short-term carry-over results. It was hypothesised that there would be no difference in the effects of 6-week SME and QSE, or 3 -month carry-over results of the 2 interventions.

\section{Material and methods}

\section{Participants}

The study was a single-blind randomized controlled trial involving patients with knee OA. All participants were volunteer male or female individuals with varying levels of knee OA severity $[9,10]$, attending the Outpatient Physiotherapy Clinic at Barau Dikko Teaching Hospital (BDTH), Kaduna, Nigeria between March 2013 and February 2015. They were diagnosed on the basis of clinical symptoms or radiographic assessment of their knee joints by orthopaedic surgeons. The subjects satisfied the American College of Rheumatology criteria for clinical classification of knee OA, i.e. pain in the knee for most days of the prior month, crepitation on active joint motion, morning stiffness less than 30 minutes in duration, patient's age 38 years or above, and bony enlargement of the knee on examination [25]. All the participants received standard medical treatment for knee OA.

Volunteers with knee OA who also had severe/uncontrolled comorbidities (such as hypertension, diabetes, kidney failure, psychiatric disorders) or a neurological condition affecting the lowerlimb (such as post-stroke muscle weakness or poliomyelitis), as well as those unable to walk were excluded from the study [26, 27]. 


\section{HUMAN MOVEMENT}

O.R. Egwu et al., Self-management in osteoarthritis

The total of 79 participants ( 66 females, 13 males) were randomized to 2 groups by having them pick a wrapped paper with A or B letter with the tag number between 1 and 100 (computer-generated random numbers) written on it from an opaque envelope. Participants who picked A were assigned to the SME group $(n=42)$ and those who picked B were assigned to the QSE group $(n=37)$.

Overall, 51 subjects $(64.6 \%)$ had bilateral knee OA, while 15 (19.0\%) had left knee OA and $13(16.4 \%)$ had right knee OA. Sixty-nine participants $(87.34 \%)$ (37 SME, 32 QSE) concluded the protocol. Among the 10 patients who did not finish the protocol, 5 (4 SME, 1 QSE) did not give relevant reasons when contacted on the telephone; 2 drop-outs (QSE) were lost because of logistic problems such as inflexibility of time and the venue of research being far from their residence; 2 (QSE) moved out of town; 1 (QSE) was hospitalised for an unknown ailment. The flowchart of the participants' recruitment and progression in the protocol is presented in Figure 1.

\section{Pain intensity assessment}

The participants were asked to identify the activity of daily living (ADL) that caused them most pain. Then, the Visual Analogue Scale (VAS) was used to assess the pain felt while performing the activity. The participant was then instructed to mark the point on the VAS that corresponded to the intensity of pain felt while performing the activity [28]. The point marked was measured with a mathematical ruler and recorded in centimetres as the participants' pain intensity. The validated Hausa version of VAS [29] was administered to the indigenous participants who could not understand the English language.

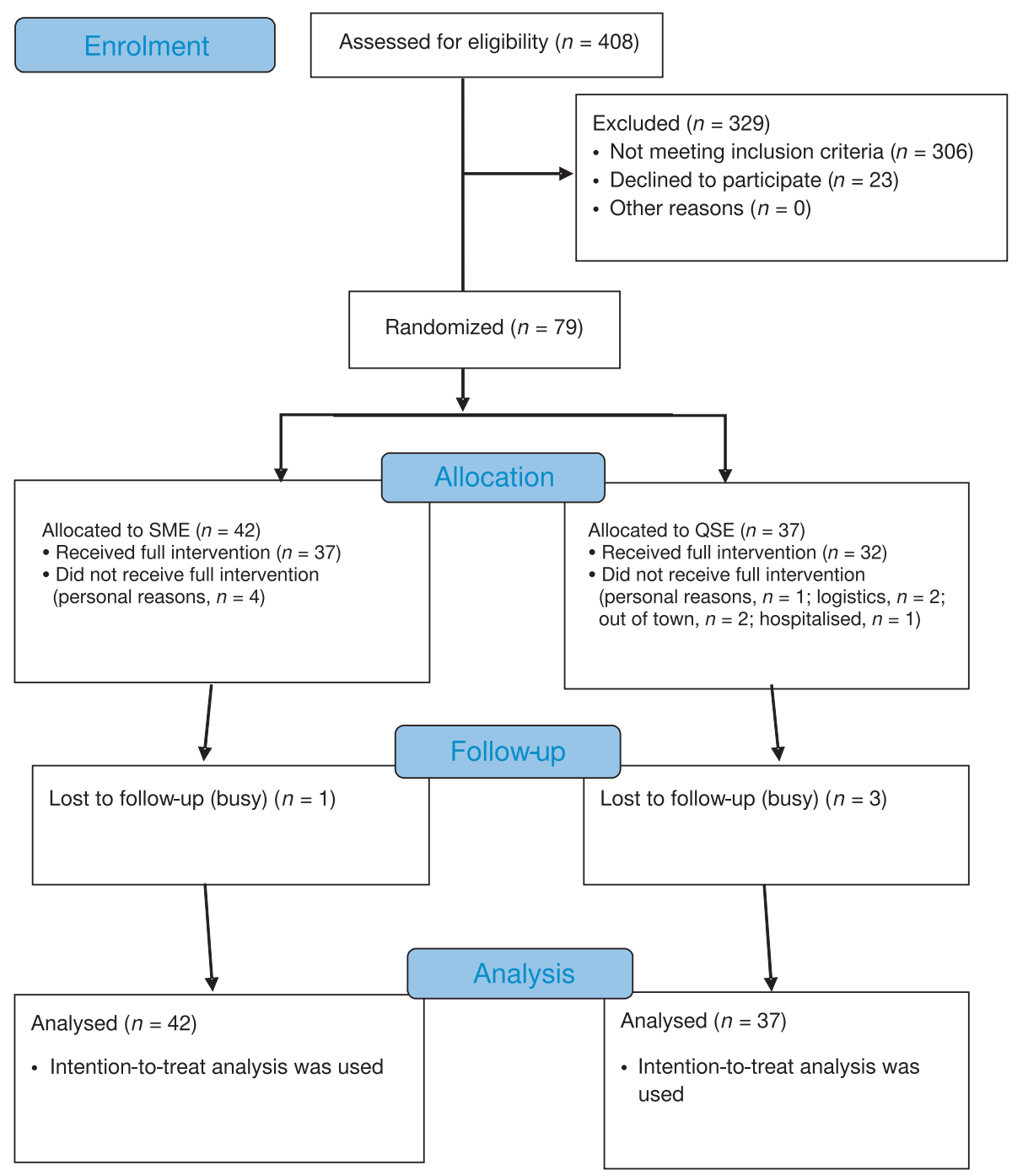

SME - self-management education, QSE - quadriceps strengthening exercise

Figure 1. Flow chart of the study 


\section{Physical function assessment}

The first section of the Ibadan Knee/Hip Osteoarthritis Outcome Measure (IKHOAM) was administered by a group-blinded assessor who also implemented the clinician's section, after which the participants performed the required physical tasks. The IKHOAM is a Nigerian culture- and environment-friendly clinical tool developed at the University of Ibadan, Nigeria for outcome assessment in the care of patients with hip or knee OA [30]. The instrument includes 3 parts, 33 items. Parts 1 and 2 are patient-reported, while part 3 is rated by the clinician. IKHOAM has been shown to demonstrate good validity and responsiveness [30, 31]. The validated Hausa version of IKHOAM [32] was administered among illiterate participants who only understood the local language. Scores were computed as follows:

- The degree of difficulty and the nature of assistance required to carry out each of the 25 items in part 1 were scored between 0 and 4 each. This gave a maximum obtainable score of $200(4 \times 25$ for degree of difficulty and $4 \times 25$ for nature of assistance).

- The extent of restriction in performing the 3 items in part 2 were scored between 0 and 3 each, giving the maximum obtainable score of $9(3 \times 3$ for extent of restriction).

- Part 3 contains 5 items with the maximum obtainable score of 23 ( 5 for 250 walk test, 4 for squat test, 5 for one-leg stance test, 4 for stairs climbing test, and 5 for balance test).

Therefore, the maximum obtainable score on IKHOAM is $232(200+9+23)$ [30].

The score for a participant was calculated in percentage as:

$$
\frac{\text { participant's score }}{\text { maximum obtainable score }} \times 100 \text { [30]. }
$$

Low and high scores on IKHOAM imply low and high levels of physical functioning, respectively [30].

Pain intensity and physical function were assessed at baseline, in week 6 , and after the $1^{\text {st }}, 2^{\text {nd }}$, and $3^{\text {rd }}$ month of follow-up.

\section{Intervention}

\section{Self-management education group}

The weekly education was conducted over a 6-week period, thereby enabling participants to incorporate and consolidate information learned from week to week.
Each weekly session comprised 2 topics and practical demonstration sessions of strengthening exercise, prevention of falls, joint protection, or thermal therapy, in line with the topic of the week. The class session lasted for 90-120 minutes. In addition to the weekly sessions, the participants were also given an information booklet on knee OA (published by Arthritis Research UK); the Hausa translated version was provided to those who did not understand English. The group size was 4-8 subjects, depending on recruitment and randomization. Each group attended the class once a week for 6 weeks. Each participant was identified by a tag number in order to monitor attendance and to ensure consistency of assessments. Self-management constructs were employed to promote behavioural changes that were aimed at optimizing the patients' health status. Goal setting and the development of strategies to achieve these goals in the long term were emphasized in the class. A lesson note was developed from the outline used by Coleman et al. [23]. A physiotherapist whose first language was Hausa was always present in class to explain the lesson for the benefit of participants who did not understand the English language.

Modules of SME adopted from Coleman et al. [23] and delivered to the participants in the SME group were focused on pathophysiology of OA, pain management strategies (cognitive, pharmaceutical, and physical), joint protection, fitness/exercise, correct use of analgesia/medications, balance, falls prevention, proprioception, team approach to health care, nutritional/ weight control, self-management skills, and specific measurable achievable realistic and time-framed goals.

\section{Quadriceps strengthening exercise group}

The participants in this group engaged in individual QSE 3 times weekly. They were instructed not to modify their ADLs and not to take part in additional forms of physical activity while the study lasted. The subjects performed the following exercises:

- Open chain quadriceps setting. The participant lay in supine position while contracting the quadriceps muscle of the affected lower extremity by drawing up the patella and maintaining the knee in extension. The contraction was held for a count of 10 , then relaxed. This was repeated 10 times [33]. The exercise was carried out by participants throughout the duration of the study [28].

- Closed chain quadriceps setting. The participant sat on a chair with their back supported, knee extended, and heel on the floor. They then pressed the heel against 
the floor and the thigh against the seat of the chair. The position was held for a count of 10 , after which the subject relaxed. This was done 10 times [33]. This exercise was carried out by the subjects throughout the duration of the study [28].

- Straight leg raising (SLR). The participant lay in supine position, contracting the quadriceps and lifting the lower extremity up to achieve about $45^{\circ}$ hip flexion while maintaining the knee in extension. This position was held for a count of 10 , then the limb was lowered. This is repeated 10 times. The contralateral knee and hip were flexed to about $45^{\circ}$ to avoid undue stress on the lower back [33]. The subjects carried out this exercise during the first 2 weeks of the study and commenced SLR with weight from the $3^{\text {rd }}$ week [28].

- Mini-squats. While standing, the participant bent both knees by about $30-60^{\circ}$, while maintaining the trunk upright. This position was maintained for a count of 10, relaxed, and repeated 10 times [33]. The subjects carried out this exercise during the first 2 weeks of the study and commenced mini-squats with weight from the $3^{\text {rd }}$ week [28]. They had barbells placed across their shoulders for mini-squats with weight [33].

Exercise progression. The participants started with a weight equivalent to their 10 repetition maximum (10-RM) and progressed with a newly determined 10-RM at the beginning of each week [32].

Determination of 10-RM. A plastic weight (W) corresponding to a certain $\mathrm{RM}$ of the participant was randomly selected and attached via the DeLorme's boot to the participant's foot. After a warm-up with a set of 10 lifts with a lighter weight, using the method described by Olagbegi et al. [27], the subjects lifted the weight R times until volitional exhaustion. The participants' 1-RM was determined with Brzycki predictive equation:

$$
1-\mathrm{RM}=\mathrm{W} /(1.0278-0.0278 \times \mathrm{R})[27]
$$

After one hour, 10-RM was determined by making the participants lift $75 \%$ of the determined 1-RM to volitional exhaustion [34]. When the subjects lifted the weight more than 10 times, the resistance was increased. If they stopped before 10 repetitions, the resistance was reduced to manage 10 lifts.

\section{Data analysis}

The data were analysed with the Statistica software package, version 13 (StatSoft, Inc, Tulsa, USA). The minimum sample size of 64 was calculated with the sample size formula by Macfarlane [35], with the effect size of 0.5 and $80 \%$ power. The effect size of 0.5 was determined in an initial pilot study with VAS as the primary outcome measure. The outcomes were analysed in accordance with the intention-to-treat principle. Information for all 79 participants was included in the analysis by carrying the last available score forward.

Descriptive statistics of mean, standard deviation, and percentages were used to summarise the data. The groups' demographic variables were compared with the independent $t$-test. With the use of treatment groups (SME and QSE) and time (baseline; week $6 ; 1^{\text {st }}, 2^{\text {nd }}$, and $3^{\text {rd }}$ month of follow-up) as between-group and within-group factors, respectively, a two-way repeated measures ANOVA was separately computed for the analysis of time, time by group interaction, and between-group effects in the intervention and follow-up phases and the entire study period. Post-hoc analysis was performed with Tukey's multiple comparison test. Cohen's rule of thumb (0.01 - small, 0.06 - moderate, 0.14 - large) was applied to categorise effect sizes (partial eta-squared), while the level of significance was set at $p<0.05$.

\section{Ethical approval}

The research related to human use has been complied with all the relevant national regulations and institutional policies, has followed the tenets of the Declaration of Helsinki, and has been approved by the Health Research Ethics Committee of the University of Ibadan and University College Hospital (Reg. No: NHREC/05/01/2008a). The permission of the management of BDTH in Kaduna, Nigeria, was also obtained.

\section{Informed consent}

Informed consent has been obtained from all individuals included in this study.

\section{Results}

Table 1 shows the demographic characteristics of the groups; there was no significant difference between the groups regarding their demographic characteristics.

The analyses of time, between-group, and time by group interaction effects for the intervention and follow-up phases and the entire duration of the study are presented in Table 2. The 2 interventions (SME and QSE) were not significantly different in their effects on pain intensity or IKHOAM scores in any of the 3 phases. During the intervention phase, there were no significant time by group interaction effects on any of the 2 outcomes; however, there were significant time effects (pain intensity: $p<0.001$, effect size $=0.603$; IKHOAM score: $p<0.001$, effect size $=0.540$ ) as pain intensity was reduced while IKHOAM score improved in the similar manner for both groups. 
Table 1. Comparison of the participants' demographics and baseline clinical variables

\begin{tabular}{|c|c|c|c|c|}
\hline \multirow[b]{2}{*}{ Variable } & \multicolumn{2}{|c|}{ Treatment groups } & \multirow[b]{2}{*}{$t$} & \multirow[b]{2}{*}{$p$-value } \\
\hline & $\begin{array}{c}\operatorname{SME}(n=42) \\
(\text { mean } \pm S D)\end{array}$ & $\begin{array}{l}\text { QSE }(n=37) \\
(\text { mean } \pm S D)\end{array}$ & & \\
\hline Age (years) & $53.86 \pm 10.40$ & $50.11 \pm 10.20$ & 1.615 & 0.110 \\
\hline Height (m) & $1.62 \pm 0.07$ & $1.62 \pm 0.07$ & -0.310 & 0.611 \\
\hline Weight (kg) & $80.0 \pm 16.98$ & $81.0 \pm 15.79$ & -0.286 & 0.776 \\
\hline BMI $\left(\mathrm{kg} / \mathrm{m}^{2}\right)$ & $31.02 \pm 6.95$ & $31.00 \pm 5.91$ & 0.012 & 0.990 \\
\hline
\end{tabular}

SME - self-management education, QSE - quadriceps strengthening exercise, BMI - body mass index

Table 2. Analysis of time, between-group, and time $\times$ group interaction effects on pain intensity and IKHOAM scores

\begin{tabular}{lrrrr}
\hline Variable/effect & $\begin{array}{c}\text { Mean } \\
\text { square }\end{array}$ & $F$ & $p$-value & $\begin{array}{c}\text { Effect } \\
\text { size }\end{array}$ \\
\hline & Intervention phase & & \\
\hline Pain intensity & & & & \\
Time & 252.769 & 116.952 & $<0.001^{*}$ & 0.603 \\
Between-group & 13.402 & 2.963 & 0.089 & 0.037 \\
Time $\times$ group & 2.440 & 1.129 & 0.291 & 0.014 \\
\hline IKHOAM scores & & & & \\
Time & 4569.200 & 90.321 & $<0.001^{*}$ & 0.540 \\
Between-group & 445.300 & 1.960 & 0.166 & 0.025 \\
Time $\times$ group & 6.500 & 0.129 & 0.720 & 0.002 \\
\hline
\end{tabular}

Follow-up phase

\begin{tabular}{|c|c|c|c|}
\hline \multicolumn{4}{|l|}{ Pain intensity } \\
\hline Time & 5.459 & 3.841 & $0.010 * 0.048$ \\
\hline Between-group & 3.991 & 0.345 & $0.559 \quad 0.004$ \\
\hline Time $\times$ group & 10.117 & 7.118 & $<0.001 * 0.085$ \\
\hline \multicolumn{4}{|l|}{ IKHOAM scores } \\
\hline Time & 317.000 & 15.627 & $<0.001 * 0.169$ \\
\hline Between-group & 49.000 & 0.114 & $0.737 \quad 0.001$ \\
\hline Time $\times$ group & 88.000 & 4.328 & $0.005 * 0.053$ \\
\hline \multicolumn{4}{|c|}{ Entire study period } \\
\hline \multicolumn{4}{|l|}{ Pain intensity } \\
\hline Time & 108.618 & 67.748 & $<0.001 * 0.468$ \\
\hline Between-group & 1.262 & 0.112 & $0.739 \quad 0.001$ \\
\hline Time $\times$ group & 8.821 & 5.502 & $<0.001 * 0.067$ \\
\hline \multicolumn{4}{|l|}{ IKHOAM scores } \\
\hline Time & 1240 & 47.010 & $<0.001 * 0.379$ \\
\hline Between-group & 147 & 0.261 & 0.6110 .003 \\
\hline Time $\times$ group & 84 & 3.197 & $0.014 * 0.040$ \\
\hline
\end{tabular}

* significance at $p<0.05$

IKHOAM - Ibadan Knee/Hip Osteoarthritis Outcome Measure
During follow-up, there were significant effects of time (pain intensity: $p=0.010$, effect size $=0.048$; IKHOAM score: $p<0.001$, effect size $=0.169)$ and time by group interaction (pain intensity: $p<0.001$, effect size $=0.085$; IKHOAM score: $p=0.005$, effect size $=0.053$ ) for both outcomes, though with small effect sizes. Pain intensity did not change in the QSE group but further decreased in the SME group as compared with the end point of the intervention phase $\left(6^{\text {th }}\right.$ week). IKHOAM scores remained the same in the SME group and decreased in the QSE group as compared with the end point of the intervention phase (6 $6^{\text {th }}$ week).

The analysis of the entire study duration indicated significant effects of time (pain intensity: $p<0.001$, effect size $=0.468$; IKHOAM score: $p<0.001$, effect size $=0.379$ ) and time by group interaction (pain intensity: $p<0.001$, effect size $=0.067$; IKHOAM score: $p=0.014$, effect size $=0.040)$ for both measures. Figures 2 and 3 illustrate time by group interaction effects and the trends of pain intensity and IKHOAM scores, respectively, across the 5 time points of the study. In

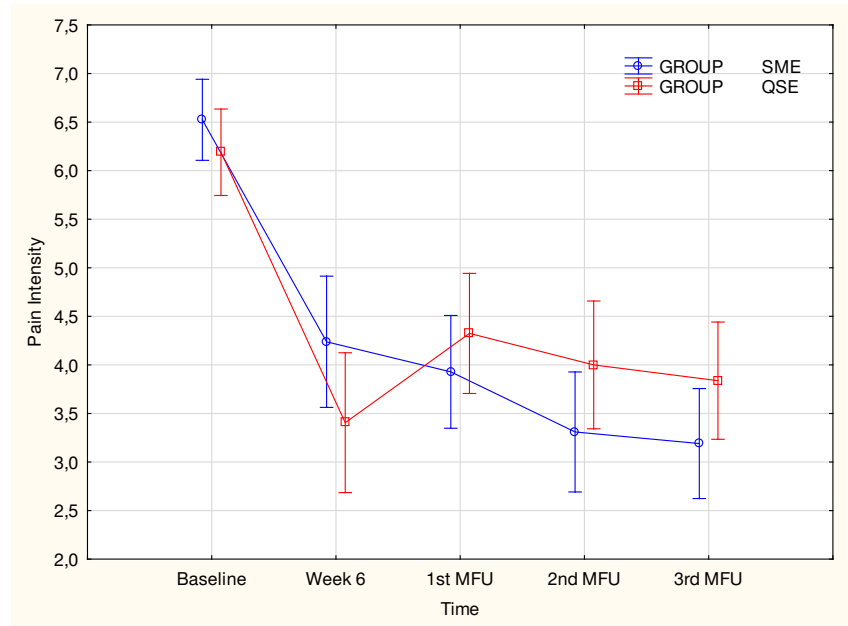

MFU - month of follow-up, SME - self-management education, QSE - quadriceps strengthening exercise

Figure 2. Trends of pain intensity for the SME and QSE group participants at the 5 time points of the study 


\section{HUMAN MOVEMENT}

O.R. Egwu et al., Self-management in osteoarthritis

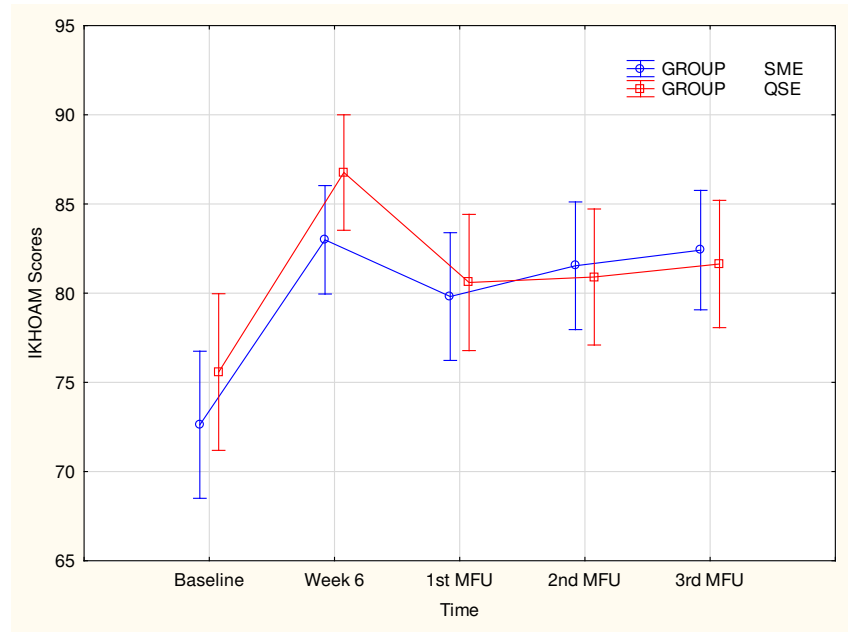

IKHOAM - Ibadan Knee/Hip Osteoarthritis Outcome Measure, MFU - month of follow-up, SME - self-management education, QSE - quadriceps strengthening exercise

Figure 3. Trends of IKHOAM scores for the SME and QSE group participants at the 5 time points of the study both groups, pain intensity at the end point of followup was significantly lower than the baseline value and IKHOAM score was significantly higher than the value obtained at the time of baseline measurements.

Post-hoc multiple comparison of changes in pain intensity and IKHOAM scores at different time intervals of the study from baseline to the end of the $3^{\text {rd }}$ month of follow-up is presented in Table 3. For the SME group, there were significant reductions in pain intensity at the time intervals of baseline/week 6 , baseline $/ 1^{\text {st }}$ month of follow-up, baseline $/ 2^{\text {nd }}$ month of follow-up, baseline $/ 3^{\text {rd }}$ month of follow-up, week $6 / 2^{\text {nd }}$ month of follow-up, and week $6 / 3^{\text {rd }}$ month of follow-up. In the QSE group, significant reductions in pain intensity were also observed at the same time intervals as in the SME group, except at week $6 / 2^{\text {nd }}$ month of follow-up and week $6 / 3^{\text {rd }}$ month of follow-up.

Both groups demonstrated a significant improvement in IKHOAM scores at baseline/week 6 , baseline $/ 1^{\text {st }}$ month of follow-up, baseline $/ 2^{\text {nd }}$ month of follow-up,

Table 3. Tukey multiple pairwise comparisons of pain intensity and IKHOAM scores for the SME and QSE groups at different time points of the study

\begin{tabular}{|c|c|c|c|c|}
\hline \multirow[b]{2}{*}{ Variable/time } & \multicolumn{2}{|l|}{ SME group } & \multicolumn{2}{|l|}{ QSE group } \\
\hline & $\begin{array}{l}\text { Mean difference } \\
(95 \% \mathrm{CI})\end{array}$ & $p$-value & $\begin{array}{l}\text { Mean difference } \\
\quad(95 \% \text { CI })\end{array}$ & $p$-value \\
\hline \multicolumn{5}{|l|}{ Pain intensity } \\
\hline BL vs. week 6 & -2.29 (from -3.33 to -1.24 ) & $<0.001^{*}$ & -2.78 (from -3.63 to -1.89 ) & $<0.001^{*}$ \\
\hline BL vs. $1^{\text {st }} \mathrm{MFU}$ & -2.60 (from -3.32 to -1.87$)$ & $<0.001^{*}$ & -1.87 (from -2.68 to -1.05 ) & $<0.001^{*}$ \\
\hline BL vs. $2^{\text {nd }} M F U$ & -3.21 (from -4.15 to -2.28 ) & $<0.001^{*}$ & -2.19 (from -3.20 to -1.80 ) & $<0.001^{*}$ \\
\hline BL vs. $3^{\text {rd }} \mathrm{MFU}$ & -3.33 (from -4.30 to -2.37$)$ & $<0.001^{*}$ & -2.35 (from -3.24 to -1.46$)$ & $<0.001^{*}$ \\
\hline Week 6 vs. $1^{\text {st }} \mathrm{MFU}$ & -0.31 (from -1.39 to 0.79 ) & 0.983 & 0.91 (from -0.02 to 1.86 ) & 0.057 \\
\hline Week 6 vs. $2^{\text {nd }} M F U$ & -0.93 (from -1.87 to 0.02 ) & $0.027 *$ & 0.60 (from -0.43 to 1.62 ) & 0.585 \\
\hline Week 6 vs. $3^{\text {rd }}$ MFU & -1.05 (from -1.94 to -0.16 ) & $0.006^{*}$ & 0.43 (from -0.56 to 1.42 ) & 0.904 \\
\hline $1^{\text {st }} M F U$ vs. $2^{\text {nd }} M F U$ & -0.62 (from -1.31 to 0.07$)$ & 0.429 & -0.32 (from -0.79 to 0.14 ) & 0.985 \\
\hline $1^{\text {st }}$ MFU vs. $3^{\text {rd }} \mathrm{MFU}$ & -0.74 (from -1.44 to -0.04$)$ & 0.185 & -0.49 (from -0.94 to -0.03 ) & 0.822 \\
\hline $2^{\text {nd }} \mathrm{MFU}$ vs. $3^{\text {rd }} \mathrm{MFU}$ & -0.12 (from -0.44 to 0.20 ) & 0.999 & -0.16 (from -0.74 to 0.41 ) & 1.000 \\
\hline \multicolumn{5}{|l|}{ IKHOAM scores } \\
\hline BL vs. week 6 & $10.37(5.56-15.17)$ & $<0.001^{*}$ & $11.19(6.50-15.87)$ & $<0.001^{*}$ \\
\hline BL vs. $1^{\text {st }} \mathrm{MFU}$ & $7.19(3.90-10.40)$ & $<0.001^{*}$ & $5.02(2.10-7.94)$ & $0.001 *$ \\
\hline BL vs. $2^{\text {nd }} M F U$ & $8.92(4.63-13.21)$ & $<0.001^{*}$ & $5.33(1.99-8.66)$ & $<0.001^{*}$ \\
\hline BL vs. $3^{\text {rd }} \mathrm{MFU}$ & $9.79(5.41-14.18)$ & $<0.001^{*}$ & $6.06(2.68-9.43)$ & $<0.001^{*}$ \\
\hline Week 6 vs. $1^{\text {st }} \mathrm{MFU}$ & -3.18 (from -7.46 to 1.10 ) & $0.010^{*}$ & -6.17 (from -10.36 to -1.97$)$ & $<0.001^{*}$ \\
\hline Week 6 vs. $2^{\text {nd }} M F U$ & -1.45 (from -5.49 to 2.58 ) & 0.955 & -5.86 (from -10.16 to -1.56$)$ & $<0.001^{*}$ \\
\hline Week 6 vs. $3^{\text {rd }} \mathrm{MFU}$ & -0.58 (from -4.20 to 3.04 ) & 1.000 & -5.13 (from -9.00 to -1.26 ) & $0.001^{*}$ \\
\hline $1^{\text {st }} M F U$ vs. $2^{\text {nd }} M F U$ & $1.73(0.06-3.39)$ & 0.876 & 0.31 (from -0.72 to 1.34 ) & 1.000 \\
\hline $1^{\text {st }}$ MFU vs. $3^{\text {rd }} \mathrm{MFU}$ & $2.60(0.72-4.49)$ & 0.375 & 1.04 (from -0.48 to 2.12 ) & 0.997 \\
\hline $2^{\text {nd }}$ MFU vs. $3^{\text {rd }} \mathrm{MFU}$ & $0.88(0.05-1.70)$ & 0.999 & 0.73 (from -0.07 to 1.53 ) & 1.000 \\
\hline
\end{tabular}

* significance at $p<0.05$

IKHOAM - Ibadan Knee/Hip Osteoarthritis Outcome Measure, SME - self-management education,

QSE - quadriceps strengthening exercise, CI - confidence interval, BL - baseline, MFU - month of follow-up 
baseline $/ 3^{\text {rd }}$ month of follow-up, week $6 / 2^{\text {nd }}$ month of follow-up, and week $6 / 3^{\text {rd }}$ month of follow-up time intervals. The QSE group significantly deteriorated in IKHOAM scores at week $6 / 1^{\text {st }}$ month of follow-up, week $6 / 2^{\text {nd }}$ month of follow-up, and week $6 / 3^{\text {rd }}$ month of follow-up time intervals; significant deterioration in IKHOAM score for SME group was only observed at the week $6 / 1^{\text {st }}$ month of follow-up time interval.

\section{Discussion}

The majority (83.54\%) of the participants in the study were female. This suggests that knee OA is more prevalent among women than men, which is consistent with previous reports in literature [23, 36]. Most of the subjects were also obese, with body mass index $(\mathrm{BMI})>30$; this appears understandable because high BMI has been associated with incidence and progression of knee OA [37].

The major aim of the study was to compare the effect of a 6-week SME and QSE intervention programmes on pain and physical function outcomes and their carry-over results in a 3-month follow-up. The 2 groups were not significantly different with regard to their pain and function outcomes at the end of the 6-week intervention. This suggests that the SME group participants achieved pain reduction and consequent functional improvement comparable with those of QSE participants, who implemented a supervised strengthening exercise programme. This may also imply that SME subjects carried out the demonstrated exercises and complied with the given instructions regarding adequate dosing and progression. Exercise has been linked to reduction in pain and improvement in function; however, it is not enough to advise a patient to exercise - it is important that the patient understands the exercise to be carried out and performs it correctly for optimal benefits [4, 38].

Although the SME and QSE interventions had statistically comparable effects on pain intensity and physical function during the follow-up phase of the study, the relationship between time and intervention depends on the values of pain intensity and IKHOAM scores, as the effects of time and group interaction were significant for both outcomes. Findings from Figures 2 and 3 suggest that pain outcome improvements achieved through SME during intervention were better sustained during the follow-up period. The participants in the SME group are likely to continue what they were taught from the educational module since they observed improvement without having to visit hospital frequently. Similar results were reported in previous studies [8, 9].
The observed increase in mean pain intensity among the QSE participants between the end of week 6 and the end of the $1^{\text {st }}$ month of follow-up (mean difference: 0.91) further suggests that the benefits of QSE are not sustainable. It is probable that a large proportion of QSE subjects did not exercise at home after the intervention; SME patients continued to demonstrate improvement in pain outcome, though with a small effect size. Roddy et al. [4], in a systematic review of homebased QSE and aerobic walking exercises, noted that adherence to exercise was a key predictor of response, and encouraging patients with knee OA to maintain exercise programmes beyond a supervised period of instruction was a major challenge. That opinion seems supported by the results of our study.

The 2 treatment programmes showed comparable significant effects on IKHOAM scores during the intervention phase of the study. This finding may be attributed to some similarities in the nature of both interventions. In the first instance, both interventions had a significant time effect on pain as observed in this study. Both included components that have been shown to directly impact reduction of pain, the leading complaint in individuals with $\mathrm{OA}$ and the one that mostly drives health care use [39]. Pain reduction has been proved directly related with reduced disability and, hence, improvement in physical function [17, 23]. Connelly et al. [39], in a recent cross-sectional survey of 197 individuals with OA, observed a strong negative correlation between physical functioning and pain.

The QSE participants, however, demonstrated inconsistent changes in physical function (IKHOAM scores) during the follow-up phase of the study. They had a markedly significant reduction (mean difference: -6.17) in their functional score at the end of the $1^{\text {st }}$ month post-intervention, which appears in line with the trend of the aforementioned changes in pain outcome. Findings from clinical trials have shown that supervised QSE improves strength [27, 40], which in turn enhances knee joint stability and function $[16,17]$. The result of the present study suggests that QSE participants were losing this clinical benefit one month post-intervention. It is, however, plausible that a number of the SME participants augmented the stability of their knees with braces and supports, since their usage was taught as part of the modules. This could be theorized to have enhanced knee joint stability, contributing to sustained function among the SME group.

Our finding with regard to physical function is consistent with the work of McKnight et al. [36], who did not observe significant differences in the effects of strength training, SME, and a combination on self-reported and 
performance-based physical function among individuals with early OA. Both studies are different in terms of the duration of supervised exercise (6 weeks vs. 9 months), outcome measures (IKHOAM vs. WOMAC), and exercise components (isometric and dynamic quadriceps strengthening vs. combination of isotonic, range of motion, stretching, and balance). Having similar results from both studies suggests that SME may confer short-term benefits regarding improvement of function for patients with knee OA.

\section{Limitations of the study}

This study is not without limitations. Analysing the effects of medical treatment that the participants received during intervention could have been helpful in interpreting the obtained results. Assessors were not blinded to the participant's intervention allocation, which could threaten the internal validity of the study. However, assessment-related bias was minimised by ensuring data recording by a neutral research assistant. Lack of a control group with knee OA undergoing no intervention is another limitation of the study. Including a control group would have shown the real treatment effects by eliminating any placebo effects produced by the intervention groups. We could not recruit controls, though, because the initial pilot study revealed that patients with knee OA attending the Physiotherapy Clinic at BDTH, Kaduna would not consent to stopping exercise for over 4 months. Severity of $\mathrm{OA}$ was also not categorized.

\section{Conclusions}

SME and supervised QSE have comparable shortterm benefits on pain and function in knee OA, but post-treatment outcome improvements appear better sustained with SME. Physiotherapists are encouraged to use the SME approach for reducing pain and disability in patients with knee OA. Incorporating physiotherapist-supervised QSE in a once-weekly SME session may enhance the effect of SME on pain and physical function. This has the potential benefit of reducing patient load in physiotherapy facilities and the financial burden of the patient because of reduced number of hospital visits.

\section{Disclosure statement}

No author has any financial interest or received any financial benefit from this research.

\section{Conflict of interest}

The authors state no conflict of interest.

\section{References}

1. Brandt KD, Dieppe P, Radin EL, Metrics P. Etiopathogenesis of osteoarthritis. Rheum Dis Clin North Am. 2008;34(3):531-559; doi: 10.1016/j.rdc.2008.05.011.

2. Çubukçu D, Sarsan A, Alkan H. Relationships between pain, function and radiographic findings in osteoarthritis of the knee: a cross-sectional study. Arthritis. 2012;984060; doi: 10.1155/2012/984060.

3. Osteoarthritis Research Society International (QARSI). Osteoarthritis: a serious disease. 2016.

4. Roddy E, Zhang W, Doherty M, Arden NK, Barlow J, Birrell F, et al. Evidence-based recommendations for the role of exercise in the management of osteoarthritis of the hip or knee - the MOVE consensus. Rheumatology. 2005;44(1):67-73; doi: 10.1093/rheumatology/ keh399.

5. Lange AK, Vanwanseele B, Fiatarone Singh MA. Strength training for treatment of osteoarthritis of the knee: a systematic review. Arthritis Rheum. 2008;59(10):14881494; doi: 10.1002/art.24118.

6. Chaipinyo K, Karoonsupcharoen O. No difference between home-based strength training and home-based balance training on pain in patients with knee osteoarthritis: a randomised trial. Aust J Physiother. 2009; 55(1):25-30; doi: 10.1016/S0004-9514(09)70057-1.

7. Bezalel T, Carmeli E, Katz-Leurer M. The effect of a group education programme on pain and function through knowledge acquisition and home-based exercise among patients with knee osteoarthritis: a parallel randomized single-blind clinical trial. Physiotherapy. 2010;96(2):137-143; doi: 10.1016/j.physio.2009. 09.009 .

8. Zhang W, Moskowitz RW, Nuki G, Abramson S, Altman RD, Arden N, et al. OARSI recommendations for the management of hip and knee osteoarthritis, part I: critical appraisal of existing treatment guidelines and systematic review of current research evidence. Osteoarthritis Cartilage. 2007;15(9):981-1000; doi: 10.1016/ j.joca.2007.06.014.

9. McAlindon TE, Bannuru RR, Sullivan MC, Arden NK, Berenbaum F, Bierma-Zeinstra SM, et al. OARSI guidelines for the non-surgical management of knee osteoarthritis. Osteoarthritis Cartilage. 2014;22(3):363388; doi: 10.1016/j.joca.2014.01.003.

10. Jordan KM, Arden NK, Doherty M, Bannwarth B, Bijlsma JWJ, Dieppe P, et al. EULAR recommendations 2003: an evidence based approach to the management of knee osteoarthritis: report of a task force of the Standing Committee for International Clinical Studies Including Therapeutic Trials (ESCISIT). Ann Rheum Dis. 2003;62(12):1145-1155; doi: 10.1136/ard.2003.011742.

11. Hippisley-Cox J, Coupland C, Logan R. Risk of adverse gastrointestinal outcomes in patients taking cyclooxygenase-2 inhibitors or conventional non-steroidal anti-inflammatory drugs: population based nested casecontrol analysis. BMJ. 2005;331(7528):1310-1316; doi: 10.1136/bmj.331.7528.1310. 
12. Hippisley-Cox J, Coupland C. Risk of myocardial infarction in patients taking cyclo-oxygenase- 2 inhibitors or conventional non-steroidal anti-inflammatory drugs: population based nested case-control analysis. BMJ. 2005;330(7504):1366-1369; doi: 10.1136/bmj.330.7504. 1366.

13. Thomas KS, Muir KR, Doherty M, Jones AC, O’Reilly SC, Bassey EJ. Home based exercise programme for knee pain and knee osteoarthritis: randomised controlled trial. BMJ. 2002;325(7367):752-755.

14. Farr JN, Going SB, McKnight PE, Kasle S, Cussler EC, Cornett M. Progressive resistance training improves overall physical activity levels in patients with early osteoarthritis of the knee: a randomized controlled trial. Phys Ther. 2010;90(3):356-366; doi: 10.2522/ ptj.20090041.

15. Hurley MV. The role of muscle weakness in the pathogenesis of osteoarthritis. Rheum Dis Clin North Am. 1999;25(2):283-298; doi: 10.1016/S0889-857X(05) 70068-5.

16. O’Reilly SC, Muir KR, Doherty M. Effectiveness of home exercise on pain and disability from osteoarthritis of the knee: a randomised controlled trial. Ann Rheum Dis. 1999;58(1):15-19; doi: 10.1136/ard.58.1.15.

17. Anwer S, Alghadir A. Effect of isometric quadriceps exercise on muscle strength, pain, and function in patients with knee osteoarthritis: a randomized controlled study. J Phys Ther Sci. 2014;26(5):745-748; doi: 10.1589/jpts.26.745.

18. Fransen M, McConnell S, Harmer AR, Van der Esch M, Simic M, Bennell KL. Exercise for osteoarthritis of the knee: a Cochrane systematic review. Br J Sports Med. 2015;49(24):1554-1557; doi: 10.1136/bjsports-2015095424 .

19. Imoto AM, Peccin MS, Trevisani VFM. Quadriceps strengthening exercises are effective in improving pain, function and quality of life in patients with osteoarthritis of the knee. Acta Orthop Bras. 2012;20(3):174179; doi: 10.1590/S1413-78522012000300008.

20. Kawasaki T, Kurosawa H, Ikeda H, Takazawa Y, Ishijima M, Kutoba M, et al. Therapeutic home exercise versus intraarticular hyaluronate injection for osteoarthritis of the knee: 6-month prospective randomized open-labeled trial. J Orthop Sci. 2009;14(2):182-191; doi: 10.1007/s00776-008-1312-9.

21. Lorig K. Partnerships between expert patient and physician. Lancet. 2002;359(9309):814-815; doi: 10.1016/ S0140-6736(02)07959-X.

22. Coleman S, Briffa K, Conroy H, Prince R, Carroll G, McQuade J. Short and medium-term effects of an education self-management program for individuals with osteoarthritis of the knee, designed and delivered by health professionals: a quality assurance study. BMC Musculoskelet Disord. 2008;9:117; doi: 10.1186/14712474-9-117.

23. Coleman S, Briffa NK, Carroll G, Inderjeeth C, Cook N, McQuade J. A randomised controlled trial of a self-man- agement education program for osteoarthritis of the knee delivered by health professionals. Arthritis Res Ther. 2012;14(1):R21; doi: 10.1186/ar3703.

24. Juhl C, Christensen R, Roos EM, Zhang W, Lund H. Impact of exercise type and dose on pain and disability in knee osteoarthritis: a systematic review and meta-regression analysis of randomized controlled trials. Arthritis Rheumatol. 2014;66(3):622-636; doi: 10.1002/ art.38290.

25. Symmons D, Mathers C, Pfleger B. Global burden of osteoarthritis in the year 2000. Global Burden Dis. 2000; 2002(4):1-26.

26. Silva LE, Valim V, Pessanha AP, Oliveira LM, Myamoto $\mathrm{S}$, Jones $\mathrm{A}$, et al. Hydrotherapy versus conventional land-based exercise for the management of patients with osteoarthritis of the knee: a randomized clinical trial. Phys Ther. 2008;88(1):12-21; doi: 10.2522/ ptj.20060040.

27. Olagbegi OM, Adegoke BO, Odole AC. Effectiveness of three modes of kinetic-chain exercises on quadriceps muscle strength and thigh girth among individuals with knee osteoarthritis. Arch Physiother. 2017;7:9; doi: 10.1186/s40945-017-0036-6.

28. Olagbegi OM, Adegoke BOA, Odole AC. Effectiveness of combined chain exercises on pain and function in patients with knee osteoarthritis. Bangladesh J Med Sci. 2016;15(2):178-188; doi: 10.3329/bjms.v15i2.24808.

29. Odole AC, Akinpelu AO. Translation and alternate forms reliability of the Visual Analogue Scale in the three major Nigerian languages. Internet J Allied Health Sci Pract. 2009;7(3):1-7.

30. Akinpelu AO, Odole AC, Adegoke BOA, Adeniyi AF. Development and initial validation of the Ibadan Knee/ Hip Osteoarthritis Outcome Measure. S Afr J Physiother. 2007;63(2):3-8; doi: 10.4102/sajp.v63i2.127.

31. Odole AC, Akinpelu AO. Translation of the Ibadan Knee/Hip Osteoarthritis Outcome Measure into Igbo language. Afr J Biomed Res. 2010;13(3):175-181.

32. Odole AC, Akinpelu AO. Validity and internal consistency of a Hausa version of the Ibadan Knee/Hip Osteoarthritis Outcome Measure. Health Qual Life Outcomes. 2008;6:86; doi: 10.1186/1477-7525-6-86.

33. Kisner C, Colby LA. Therapeutic exercise: foundations and techniques. Philadelphia: F.A. Davis Company; 2007.

34. Aarskog R, Wisnes A, Wilhelmsen K, Skogen A, Bjordal JM. Comparison of two resistance training protocols, $6 \mathrm{RM}$ versus $12 \mathrm{RM}$, to increase the $1 \mathrm{RM}$ in healthy young adults. A single-blind, randomized controlled trial. Physiother Res Int. 2012;17(3):179-186; doi: 10.1002/pri.527.

35. Macfarlane TV. Sample size determination for research projects. J Orthod. 2003;30(2):99-100; doi: 10.1093/ ortho/30.2.99.

36. McKnight PE, Kasle S, Going S, Villaneuva I, Cornett M, Farr J, et al. A comparison of strength training, selfmanagement and the combination for early osteoar- 


\section{HUMAN MOVEMENT}

O.R. Egwu et al., Self-management in osteoarthritis

thritis of the knee. Arthritis Care Res. 2010;62(1):4553; doi: 10.1002/acr.20013.

37. Zeni JA, Snyder-Mackler L. Most patients gain weight in the 2 years after total knee arthroplasty: comparison to a healthy control group. Osteoarthritis Cartilage. 2010;18(4):510-514; doi: 10.1016/j.joca.2009.12.005.

38. Cottrell E, Roddy E, Foster NE. The attitudes, beliefs and behaviours of GPs regarding exercise for chronic knee pain: a systematic review. BMC Fam Pract. 2010; 11:4; doi: 10.1186/1471-2296-11-4.

39. Connelly AE, Tucker AJ, Kott LS, Wright AJ, Duncan AM. Modifiable lifestyle factors are associated with lower pain levels in adults with knee osteoarthritis. Pain Res Manag. 2015;20(5):241-248; doi: 10.1155/ 2015/389084.

40. Jan MH, Lin CH, Lin YF, Lin JJ, Lin DH. Effects of weight-bearing versus nonweight-bearing exercise on function, walking speed, and position sense in participants with knee osteoarthritis: a randomized controlled trial. Arch Phys Med Rehabil. 2009;90(6):897-904; doi: 10.1016/j.apmr.2008.11.018. 